\title{
Corela
}

Cognition, représentation, langage

18-1 | 2020

Vol. $18, n^{\circ} 1$

\section{Introduction du volume}

\section{Sylvester Osu}

\section{(2) OpenEdition}

Journals

Édition électronique

URL : http://journals.openedition.org/corela/11446

DOI : $10.4000 /$ corela. 11446

ISSN : 1638-573X

Éditeur

Cercle linguistique du Centre et de l'Ouest - CerLICO

\section{Référence électronique}

Sylvester Osu, «Introduction du volume », Corela [En ligne], 18-1 | 2020, mis en ligne le 29 juin 2020, consulté le 01 juillet 2020. URL : http://journals.openedition.org/corela/11446 ; DOI : https://doi.org/ 10.4000/corela.11446

Ce document a été généré automatiquement le 1 juillet 2020.

\section{(c) (i) (2) (2)}

Corela - cognition, représentation, langage est mis à disposition selon les termes de la licence Creative Commons Attribution - Pas d'Utilisation Commerciale - Partage dans les Mêmes Conditions 4.0 International. 


\section{Introduction du volume}

\section{Sylvester Osu}

1 Ce numéro (18.1 de CORELA) propose trois articles. Le premier article de Nathalie Garric, Frédéric Pugnière-Saavedra et Valérie Rochaix intitulé "Construction langagière de la figure de l'aidant du malade d'Alzheimer : dénominations et mise en mots interdiscursive dans les pratiques" s'intéresse au statut de l'aidant dans l'accompagnement non professionnel de patients atteints de la maladie d'Alzheimer. L'objectif est donc de contribuer à la compréhension d'une problématique urgente, celle de l'accompagnement de la fin de vie. Se plaçant dans une perspective des sciences du langage, les auteurs envisagent le rôle de cette maladie et la fonction du proche impliqué dans une relation d'aide ainsi que leur construction sociale, politique et sanitaire. L'article rappelle le rôle crucial de la reconnaissance juridique de l'aidant puis, présente la figure de l'aidant sur le terrain de la recherche y compris en sciences $\mathrm{du}$ langage où les travaux se centrent prioritairement sur les caractéristiques du discours de patients atteints de la maladie d'Alzheimer ou, dans une perspective sociolinguistique et/ou conversationnelle, sur la relation soignant/patient. Et s'inscrivant dans le cadre de l'analyse du discours, et de façon complémentaire sur une méthodologie en corpus qualitative (Sémantique des Possibles Argumentatifs et analyse énonciative indicielle) et quantitative appuyée sur la lexicométrie à l'aide du logiciel Lexico, l'article vise une meilleure compréhension de l'aidance et de ses enjeux en étudiant comment ces derniers sont construits dans la circulation des points de vue qui s'expriment (discours et contre-discours), sans s'en tenir aux représentations dominantes, de la doxa ${ }^{1}$ médiatique ou de communautés légitimes, celle du discours juridique.

2 S'appuyant sur des données recueillies lors de ses différentes missions de terrain effectuées entre 2016 et 2018 à Ziguinchor (Sénégal) et en Guinée-Bissau, Dame Ndao "L'intégration morphologique des emprunts créoles dans la langue mancagne " s'intéresse à la manière par laquelle la langue mancagne, une langue à classes nominales (atlantique, Niger-Congo, parlée au Sénégal et en Guinée-Bissau) s'approprie et intègre dans son lexique les mots empruntés au créole de Bissau. Il constate que l'intégration de ces emprunts ne se fait pas de manière libre mais au contraire, obéit à des règles différentes. L'auteur distingue alors trois cas de figures: 1) si le mot 
comporte, à l'initiale, une séquence susceptible d'être interprétée par les locuteurs du mancagne comme un préfixe de classe, il sera affecté à la classe correspondante ;2) si on ne peut pas avoir recours à la forme du mot emprunté pour lui attribuer une classe, le mot est affecté aux classes u- ou p- (singulier) et i (pluriel) ; 3) si la syllabe initiale ne correspond à aucun préfixe de classe de la langue, le mancagne l'affecte à la classe $u$-.

L'article de Michèle Debrenne fait un état des lieux des dictionnaires d'associations évoquées par les mots en tant qu'ils reflètent le lien entre le lexique et l'image du monde. L'auteure rappelle que ces dictionnaires se présentent sous deux formes: le dictionnaire direct, comportant les réactions classées par ordre de fréquence, et le dictionnaire inverse, dans lequel le mot vedette est la réaction, couplée aux stimuli qui l'ont provoquée. L'approche consiste à appliquer la méthode psycholinguistique de fixation de la première réponse à un stimulus lexical. Plus précisément, cet article présente les principes sur lesquels ces dictionnaires sont construits, la méthodologie, les résultats et leur exploitation. Ainsi, après quelques repères historiques, il aborde d'abord les dictionnaires français d'associations évoquées par les mots. Cette partie est illustrée à travers une discussion sur le dictionnaire des associations verbales du français (DAF) d'une part, et sur le dictionnaire des normes associatives de la Francophonie (DINAF) d'autre part. L'article aborde ensuite l'exploitation des données des dictionnaires d'associations évoquées par les mots et s'intéresse en particulier à l'analyse du champ associatif où champ associatif renvoie à l'ensemble structuré des réactions obtenues à un stimulus, tirées de l'article du dictionnaire direct, puis à l'analyse des données du dictionnaire inverse (les superconnecteurs) qui permet d'établir la liste des réactions liées au plus grand nombre de stimuli. Il aborde enfin les applications de ces dictionnaires d'associations évoquées par les mots dans le domaine de l'enseignement du FLE. Ainsi par exemple, l'analyse du réseau associatif verbal révélé par le dictionnaire inverse peut permettre de compléter l'information fournie par un dictionnaire de fréquence pour repérer les mots à enseigner en premier lieu. Ou encore, l'analyse avec les apprenants du contenu sémantique d'une notion et surtout la charge culturelle partagée.

\section{NOTES}

1. Nous concevons la doxa dans le cadre de la théorie générale du sens commun de Sarfati, où elle constitue un cadre idéologique tel qu'il est construit par les discours et s'envisage de façon plurielle, chaque champ de pratiques discursives définissant sa propre doxa : elle renvoie «aux poncifs, stéréotypes, lieux communs que les locuteurs convoquent avec «une relative méconnaissance de ce qu'ils sont » (Sarfati, $1988: 22-23)$. 
AUTEUR

SYLVESTER OSU

Université de Tours/LLL 